\title{
ON THE ROLE OF STAR CATALOGUES FOR AUTONOMOUS SPACE NAVIGATION
}

\author{
V. V. IVASHKIN \\ Keldysh Institute of Applied Mathematics \\ Miusskaya Sq., 4, 125047 Moscow \\ USSR
}

\begin{abstract}
The questions connected with constructing modern systems of autonomous navigation for spacecraft are considered. Increasing importance of these systems is shown, which is due to: a sharp increase in a number of spacecraft launches and congestion of on-ground control centers; and a necessity to provide more accurate and timely determination of motion parameters and motion control of spacecraft during their flights to the Solar System's celestial bodies. Importance of the star sighting and angular measurements connected with stars for adequate solution of space navigation problems is shown. Thus the star catalogues play a significant role for constructing navigational algorithms.
\end{abstract}

1. INTRODUCTION. An autonomous system of space navigation allows some (optical,for example) on-board measurements of observed parameters, their statistical processing and determining the spacecraft motion parameters. Importance of these systems was already noted by pioneers of cosmonautics. With development of practical cosmonautics such systems were implemented in spacecraft designs since they sometimes allowed determining the spacecraft motion and orientation more timely and accurately, especially in situations of emergency and in transfers to the Solar System planets.For example, for decelerating the Luna-9 station to make a soft landing onto the Moon, by using an autonomous optical system the station velocity vector direction was very accurately determined and then reserved. Autonomous determination of the spacecraft orbit was provided in the Apollo Project. With time the autonomous navigation systems become more vital, even for artificial satellites.

\section{STELLAR MEASURRMENTS IN AUTONOMOUS NAVIGATION}

Astronomical navigation is a most important form of autonomous navigation. It is based on optical measurements of some parameters. As a rule, stars are main objects for observation here. They participate in navigation either directly or indirectly - allowing the construction of an onboard inertial coordinate system (ICS), in which the directions to 
other celestial bodies (planets, the Sun) are then determined.

Now, the optical autonomous methods are gaining wide acceptance even in the near-earth orbital flights because potentially they allow constructing a fully autonomous and sufficiently accurate navigation system, which can help to relieve onground control centers. It is most promising to use the measurements of such parameters as angles of star elevations over horizon, the times of star set and rise above the planet horizon, angles of star refraction in the planetary atmosphere, angles of sighting line orientation relatively ICS for landmarks on the planet's surface as is shown in [1-3]. Such navigation allows determining the spacecraft position relatively a planet within $1 \mathrm{~km}$ or better.

In interplanetary transfers the main autonomous measurements are the angles of the orientation of sighting lines of closest celestial bodies relatively ICS. For distances about $100 \mathrm{million} \mathrm{km}$ to celestial bodies and accuracies of angular measurements about 1 arcsec the spacecraft position can be determined within a few hundreds kilometers, which is a satisfactory back-up to the ground measurements (especially in the emergency) for the middle part of flight to other planet.

The autonomous optical navigation is particularly important in the mission of rendezvous with a remote planet due to large time lags in communication with the spacecraft and an uncertainty in knowledge of the planet motion. In the framework of the Soviet-French Vesta Project in [4] the study of such navigation was carried out for transfer to an asteroid.Optical-TV sighting of the planet against the star background and computer processing of obtained measurements, characterizing the orientation of the planet's sighting line in ICS, allow good determination of the spacecraft's and landing probe's motion relatively the asteroid (within 10 to $25 \mathrm{~km}$ ) and correction of knowledge on the asteroid position.

3.CONCLUSIONS. In modern cosmonautics the autonomous optical measurements using the stars are of great importance, and in future, as the problems to be solved will grow in complexity, their role will become even more and more significant. Therefore, the star catalogues which give a basis for using the star characteristics in navigational algorithms are very important for guidance, navigation and control of spacecraft.

\section{REFERRENCES}

1. Battin, R.H. (1964) Astronautical Guidance, MoGraw-Hill Book Company, Inc., New York.

2. Gounley, R., White, R., and Gai,E. (1984) 'Autonomous satellite navigation by stellar refraction ", Joumal of Guidance, Control and Dynamics 2, 129-134.

3. Levine, G.M. (1966) 'A method of orbital navigation using optical sighting to unknown landmarks", AIAA Journal 4,1928-1931.

4. Ivashkin, V.V. (1988) Navigation analysis of the space probe for investigation of the Vesta asteroid ', Preprint, Keldysh Institute of Applied Mathematics, USSR Ac. Sci., N152. 